\title{
Molecular Hydrogen Production from Amorphous Solid Water during Low Energy Electron Irradiation
}

\author{
Kamel A. K. Gadallah ${ }^{1,2,}{ }^{*}$, Demian Marchione ${ }^{1,3}$, Sven P. K. Koehler ${ }^{4,5}$ \\ and Martin R. S. McCoustra ${ }^{1}$ \\ ${ }^{1}$ School of Engineering and Physical Sciences, Heriot-Watt University, Riccarton, Edinburgh EH14 4AS, UK. \\ ${ }^{2}$ Astronomy \& Meteorology Department, Faculty of Science, Al-Azhar University, Nasr City, PO Box 11884, \\ Cairo, Egypt. \\ ${ }^{3}$ Science Division, Jet Propulsion Laboratory, California Institute of Technology, Pasadena, CA 91109, USA. \\ ${ }^{4}$ School of Chemistry, The University of Manchester, Manchester, M13 9PL, UK. \\ ${ }^{5}$ Dalton Cumbrian Facility, The University of Manchester, Westlakes Science \& Technology Park, Moor Row, \\ CA24 3HA, UK
}

* Corresponding Author email: Kamel.Gadallah71@Gmail.com

\begin{abstract}
This work investigates the production of molecular hydrogen isotopologues $\left(H_{2}, H D\right.$, and $\left.D_{2}\right)$ during low energy electron irradiation of layered and isotopically labelled thin films of amorphous solid water (ASW) in ultrahigh vacuum. Experimentally, the production of these molecules with both irradiation time and incident electron energy in the range 400 to 500 $\mathrm{eV}$ is reported as a function of the depth of a buried $\mathrm{D}_{2} \mathrm{O}$ layer in an $\mathrm{H}_{2} \mathrm{O}$ film. $\mathrm{H}_{2}$ is produced consistently in all measurements, reflecting the $\mathrm{H}_{2} \mathrm{O}$ component of the film, though it does exhibit a modest reduction in intensity at the time corresponding to product escape from the buried $D_{2} \mathrm{O}$ layer. In contrast, $H D$ and $D_{2}$ production exhibit peaks at times corresponding to product escape from the buried $D_{2} \mathrm{O}$ layer in the composite film. These features broaden the deeper the $H D$ or $D_{2}$ is formed due to diffusion. A simple random-walk model is presented that can qualitatively explain the appearance profile of these peaks as a function of the incident electron penetration.
\end{abstract}

* Corresponding author 


\section{Introduction}

Characterisation of amorphous solid water (ASW) is of general interest in surface chemistry ${ }^{1-5}$, but especially relevant to astrophysics and planetary science where solid water is an important component of comets $^{6}$, planetary satellites ${ }^{7,8}$, and the icy mantles that coat interstellar dust grains ${ }^{9-11}$. The surface chemistry of nano- to micrometre-sized particles plays an important role in molecular synthesis in interstellar space ${ }^{12}$. In cold, dense molecular clouds, dust grain particles are covered by icy mantles that contain mainly $\mathrm{H}_{2} \mathrm{O}$ in combination with minor quantities of other molecules such as $\mathrm{CO}, \mathrm{CO}_{2}, \mathrm{NH}_{3}$, and $\mathrm{CH}_{4}{ }^{13-21}$. Starting from these relatively simple species, thermal and non-thermal processing of icy dust grains in the interstellar medium (ISM) significantly contributes to the observed abundance of complex organic molecules (COMs) ${ }^{12,22 .}$

The presence of both $H$ and $D$ atoms in the interstellar medium (ISM) means that deuteration reactions can occur on grain surfaces; deuterated products are then observed when they are released in to the gas phase ${ }^{23,}{ }^{24}$. IR observations have detected $D_{2} \mathrm{O}$ in the ISM toward warm regions where water ices have evaporated ${ }^{25,}{ }^{26}$. Kristensen et al. ${ }^{27}$ estimated the abundances of $H_{2}, H D$, and $D_{2}$ on ice-covered dust grains in dark clouds, showing that $D_{2}$ is the most abundant deuterated molecule on the grain surface at $10 \mathrm{~K}$ whereas HD is more abundant in the gas phase. Indeed the $\mathrm{D} / \mathrm{H}$ ratio in cold dense objects can reach of the order of $0.1-0.2$ compared to the average of $1.5 \times 10^{-5}$ in the galactic interstellar medium ${ }^{28,29}$. Recent studies have reported the degree of deuterium enrichment and fractionation in many stellar objects at different evolutionary stages ${ }^{30-41}$. Fontani et al. ${ }^{42}$ show that surface chemistry has an important role in determining the fractional deuteration of some molecules (e.g. $\mathrm{NH}_{3}$ and $\mathrm{CH}_{3} \mathrm{OH}$ ) formed on the interstellar grain mantles. This study confirmed that the deuterated fractions of $\mathrm{N}_{2} \mathrm{H}^{+}$and $\mathrm{CH}_{3} \mathrm{OH}$, respectively, are good tracers of massive starless cores and early proto-stellar phases, where the evaporation or sputtering of the grain mantles occurs most efficiently ${ }^{42}$.

As the dominant solid in the Universe; as the solvent upon which biology depends; and as a common solvent in radiological environments, it is crucial to understand the interaction of $\mathrm{H}_{2} \mathrm{O}$ with ionising radiation ${ }^{43-45}$. In ultrahigh vacuum (UHV), radiolysis of ASW thin films 
results in both desorption of $\mathrm{H}_{2} \mathrm{O}$, as well as a range of neutral and charged species, and chemical reactions. These processes are promoted by the large number of low-energy secondary electrons generated in the ASW film by the passage of the primary charged particles. As a consequence of their large scattering cross sections and short mean-free paths, these secondary electrons promote a variety of chemical and physical processes in condensed $\mathrm{H}_{2} \mathrm{O}$ which have been extensively investigated ${ }^{1,46-50}$. While Petrik and Kimmel ${ }^{1}$ highlight the nature of electronic excitations in $\mathrm{H}_{2} \mathrm{O}$ that determine its radiolysis behaviour, others focus more closely on the chemical reactions promoted by low energy electrons ${ }^{46,47}$, 51, 52. Petrik et al.5, 53, 54 have reported on low-energy electron irradiation of ASW films, deuterated ASW ices, and other similar systems including buried CO layers. The focus of that work is set on the formation and subsequent desorption of volatile species, such as molecular hydrogen $\left(\mathrm{H}_{2}\right.$ or $\left.\mathrm{D}_{2}\right)$ or oxygen $\left(\mathrm{O}_{2}\right)$, which mainly occurs at the vacuum interface and within the first monolayers or at the substrate/ASW interface, but not in the bulk where reactions of the primary products of radiolysis are important.

In this work, we aim to study the electron-stimulated production and desorption of $\mathrm{H}_{2}, \mathrm{HD}$ and $\mathrm{D}_{2}$ molecules from layered $\left(\mathrm{H}_{2} \mathrm{O} / \mathrm{D}_{2} \mathrm{O} / \mathrm{H}_{2} \mathrm{O}\right)$ thin films of $A S W$, as a function of both the depth of the buried $D_{2} \mathrm{O}$ layer from the vacuum interface as well as the energy of the incident electrons. The CASINO (the monte Carlo Simulation of electron trajectory in sOlids) $\operatorname{code}^{55}$ has been used to predict the distribution of electron penetration depths as a function of the ice composition and electron energy (300 eV - $1000 \mathrm{eV}$ ). In addition, a simple Monte Carlo model based on the work of Petrik et $a .^{5}$ has been employed to elucidate the physical and chemical processes occurring in these systems.

\section{Methodology}

\section{Experiment}

The experimental apparatus, a UHV chamber with a base pressure of $2 \times 10^{-10}$ Torr, has been described in detail elsewhere ${ }^{56-59}$. Hence, only a brief description will be given here. Samples of layered films of ASW were deposited on a polished stainless steel disc (10 mm diameter and $2 \mathrm{~mm}$ thickness) at a base temperature of $\sim 108 \mathrm{~K}$, by background dosing ${ }^{60,} 61$ from two 
independent gas manifolds containing $\mathrm{H}_{2} \mathrm{O}$ and $\mathrm{D}_{2} \mathrm{O}$ (purities of $99.9 \%$ and $99.9 \%$, respectively; Sigma-Aldrich). The chamber was evacuated using a liquid nitrogen trapped 9" oil diffusion pump, a small $8 \mathrm{dm}^{3} \mathrm{~s}^{-1}$ diode ion pump (Varian Vaclon), and a titanium sublimation pump (Leisk Engineering). The substrate was cooled to base temperature by continuously filling a liquid nitrogen reservoir, which is thermally connected to the stainless steel disc. Electron irradiation was carried out using electrons at energies in the range 400 to $500 \mathrm{eV}$, from a low-energy electron gun (Kimball Physics ELG-2), incident at an angle of $30^{\circ}$ with respect to the surface normal ${ }^{58,59}$. Typical beam currents on the sample were measured using a homebuilt picoammeter directly connected to the substrate. Desorption profiles of species evolved produced during the irradiation were recorded using a quadrupole mass spectrometer (QMS, VG Microtech PC300D) facing the front of the sample fitted with a homemade line-of-sight tube.

$\mathrm{H}_{2} \mathrm{O}$ and $\mathrm{D}_{2} \mathrm{O}$ liquids were de-gassed and purified by multiple freeze-pump-thaw cycles. Layered icy ASW films were deposited by dosing separately $\mathrm{H}_{2} \mathrm{O}$ and $\mathrm{D}_{2} \mathrm{O}$ through the gas manifolds resulting in a total exposure of $400 \mathrm{~L}$ (where $1 \mathrm{~L}$ is equivalent to $10^{-6}$ Torr $\mathrm{s}$ uncorrected for the ionisation coefficient). We would like to stress that while dosing even at pressures of $10^{-6}$ Torr the heat release on condensation is rapidly removed by the refrigeration and has no significant impact on the structure of our compact ASW films. After correcting for the ionization gauge sensitivity $\left(1.1\right.$ for $\left.\mathrm{H}_{2} \mathrm{O}^{62}\right)$, the total exposure becomes $363 \mathrm{~L}$. The relationship between film thickness and exposure is assumed to be linear for both pure $\mathrm{H}_{2} \mathrm{O}$ and $\mathrm{D}_{2} \mathrm{O}$ ices. The film thickness corresponding to this exposure has been calculated to be $44.7 \mathrm{~nm}$ for pure $\mathrm{H}_{2} \mathrm{O}$ assuming that the molecular density and deposition rate are homogeneous in time and space over the substrate. Fundamental to this calculation is the assumption that the sticking coefficients of $\mathrm{H}_{2} \mathrm{O}$ and $\mathrm{D}_{2} \mathrm{O}$ are unity at the experimental temperature.

Figure 1 schematically represents the structure of the ASW film while Table 1 summarises the details of that structure. All samples were irradiated for 10 minutes with an electron beam with a beam cross sectional area of approximately $1 \mathrm{~mm}^{2}$ using a typical beam current of $1.725 \mu \mathrm{A}$ corresponding to an electron flux of $(1.08 \pm 0.03) \times 10^{15}$ electrons $\mathrm{cm}^{-2} \mathrm{~s}^{-1} . \mathrm{H}_{2}(\mathrm{~m} / \mathrm{z}$ 
$=2), H D(m / z=3)$ and $D_{2}(m / z=4)$ desorbing from the icy film were monitored using the QMS operating in analogue mode. The resulting mass signal versus time was then baselinecorrected.

\section{Simulation}

To understand the possible physio-chemical processes occurring during the electron irradiation, it is important to estimate the penetration depth of the incident electrons into the icy ASW films. Figure 2 displays the intensity ratio of the transmitted electron beam with respect to the incident beam as a function of the film thickness of pure amorphous $\mathrm{H}_{2} \mathrm{O}$ ice. Each curve has been estimated taking into account the energy dependent mean free path of the electrons ${ }^{63}$. The intensity of the electron beam decreases exponentially with the penetration.

Version 2 of the CASINO code ${ }^{55}$ was used to simulate the penetration of primary electrons within the multi-layered $\mathrm{H}_{2} \mathrm{O} / \mathrm{D}_{2} \mathrm{O} / \mathrm{H}_{2} \mathrm{O}$ ASW thin films using Monte Carlo methods. The films were assumed to have a total thickness of $44 \mathrm{~nm}$ with a very thin layer $(0.23 \mathrm{~nm})$ of $\mathrm{D}_{2} \mathrm{O}$ buried at different depths inside the sample. The density of vapour deposited $\mathrm{H}_{2} \mathrm{O}$ ice from a molecular beam varies from $0.82 \mathrm{~g} \mathrm{~cm}^{-3}$ at $90 \mathrm{~K}$ to $0.93 \mathrm{~g} \mathrm{~cm}^{-3}$ at $130 \mathrm{~K}^{60,64}$. By interpolation, the density of condensed water ice at $108 \mathrm{~K}$ is $0.86 \mathrm{~g} \mathrm{~cm}^{-3}$. For $\mathrm{D}_{2} \mathrm{O}$ ice, we use the ratio between liquid and solid $\mathrm{H}_{2} \mathrm{O}$ densities as scaling factor to estimate the solid $\mathrm{D}_{2} \mathrm{O}$ density $\left(0.96 \mathrm{~g} \mathrm{~cm}^{-3}\right)$. The distribution of the primary electrons was simulated for a low-energy electron beam incident on the sample at $30^{\circ}$ and it is found to be a function of both the film thickness and electron energy. These simulations assume a Gaussian profile for the electron beam propagating through the ASW layer and enumerate the number of electron trajectories that are not back scattered out of the solid as a function of penetration depth.

\section{Results}

\section{Experiment}

The desorption profiles of $\mathrm{H}_{2}, \mathrm{HD}$ and $\mathrm{D}_{2}$ resulting from electron-induced chemistry (EIC) in the layered ASW films are shown in Figure 3. Remarkably, the latter two species result from 
EIC within and adjacent to the $D_{2} \mathrm{O}$ layer at the vacuum interface or buried between $2 \mathrm{~nm}$ and $11 \mathrm{~nm}$ below the vacuum interface when these samples are exposed to $450 \mathrm{eV}$ electrons. Figure 3(a) reports the yield of $\mathrm{H}_{2}$ for samples $\mathrm{S} 1$ to $\mathrm{S} 5$ listed in Table 1 as the $\mathrm{H}_{2} \mathrm{O}$ cap layer thickness is increased from 0 to approximately $11 \mathrm{~nm}$. Figure $\mathbf{3}(\mathbf{b})$ and (c) present the yields of $H D$ and $D_{2}$, which appear at increasingly longer times as the depth of the $D_{2} O$ layer is increased. It appears that the arrival times of $H D$ and $D_{2}$ are a function of the diffusion length through the $\mathrm{H}_{2} \mathrm{O}$ cap layer. It is noticeable that the peak value observed for each of the HD and $D_{2}$ yields appears to match a corresponding dip in the $\mathrm{H}_{2}$ yield. This behaviour can be explained by the effective absence of $\mathrm{H}_{2} \mathrm{O}$ (and hence $\mathrm{H}_{2}$ production) in the $\mathrm{D}_{2} \mathrm{O}$ layers.

This behaviour reflects the variation in the $\mathrm{H}_{2} \mathrm{O} / \mathrm{D}_{2} \mathrm{O}$ destruction rates, the $\mathrm{H}_{2}, \mathrm{HD}$ and $\mathrm{D}_{2}$ formation rates, and the diffusion of these species and their atomic progenitors in the ASW film. The former, because of their electron-promotion, will be sensitive functions of electron penetration depth and secondary electron formation. Sample S1 is the only ice without a capping layer of $\mathrm{H}_{2} \mathrm{O}$ leaving $\mathrm{D}_{2} \mathrm{O}$ exposed at the vacuum interface. Therefore, both $\mathrm{D}_{2}$ and HD desorption profiles display a sharp peak as soon as irradiation begins, whereas a delayed peak is observed in the $\mathrm{H}_{2}$ trace as $\mathrm{H}_{2}$ is clearly only produced in the $\mathrm{H}_{2} \mathrm{O}$ layer underneath the $\mathrm{D}_{2} \mathrm{O}$ (indicating that isotope exchange is not overly pronounced under our experimental conditions). The corresponding slow appearance of $\mathrm{H}_{2}$ in this trace reflects the combination of the rates of $\mathrm{H}_{2}$ formation and its diffusion to the vacuum interface (See Figure 3 (a); $\mathrm{S} 1$ ).

Figure 4 illustrates the $H_{2}, H D$ and $D_{2}$ formation/desorption yields from identical $\mathrm{H}_{2} \mathrm{O} / \mathrm{D}_{2} \mathrm{O} / \mathrm{H}_{2} \mathrm{O}$ ASW films irradiated with 400,450 , and $500 \mathrm{eV}$ electrons, respectively. These samples have the same overall thickness of $44.7 \mathrm{~nm}$, with the $\mathrm{D}_{2} \mathrm{O}$ layer buried at a constant depth of $5.4 \mathrm{~nm}$. Although the thickness and arrangement of the layered ices in this series of experiments is fixed, the desorption profiles of $H_{2}, H D$ and $D_{2}$ in Figure 4 appear to be sensitive to the energy of the primary electrons. In particular, the HD and $D_{2}$ peaks (Figure 4b and 4c) appear earlier and narrower when the electron energy is highest (500 eV), whereas the curves corresponding to lower electron energy experiments (e.g. $400 \mathrm{eV}$ ) appear later and show broader features. Remarkably, in the case of $500 \mathrm{eV}$ electrons, we 
note a sudden increase in the desorption profiles of the observed molecular species in the first $70 \mathrm{~s}$ of irradiation.

Furthermore, the slightly decreasing background in Figure $\mathbf{3}$ and $\mathbf{4}$ may be due to a decrease in precursor concentration as fewer intact water molecules are available over time. When an $\mathrm{H}_{2} \mathrm{O}$ cap layer (2.6 to $11.0 \mathrm{~nm}$ thick, Table 1 ) is added on top of the $\mathrm{D}_{2} \mathrm{O}$ ice, $\mathrm{H}_{2}$ appears as soon as the electron beam is incident on the ASW. The HD and $D_{2}$ peaks are delayed and broadened with increasing depth of the $D_{2} \mathrm{O}$ layer, suggesting that the detected molecular species are formed within the bulk.

\section{Simulation}

The CASINO codes provides simulations of electron trajectories as a function of the penetration depth along the direction normal to the film surface assuming a Gaussianshaped electron beam travelling through the ice. Figure $\mathbf{5}$ shows the distribution of electron trajectories in normal to the surface (the z-direction) as a function of the penetration depth for multi-layered ices consistent with those investigated experimentally. The figure shows that, for $450 \mathrm{eV}$ electrons, there is no penetration beyond $18 \mathrm{~nm}$. Given the films are 44.7 $\mathrm{nm}$ thick, this implies that all the incident electrons are stopped within the compact ASW film. None of the $450 \mathrm{eV}$ primary electrons reach the underlying stainless steel substrate and so we can assume that there is no significant electron-promoted chemistry at the metal-ASW interface. The calculations highlight that for all of our experiments, the $450 \mathrm{eV}$ primary electrons can reach the $\mathrm{D}_{2} \mathrm{O}$ inter-layer in the multi-layered $\mathrm{H}_{2} \mathrm{O} / \mathrm{D}_{2} \mathrm{O} / \mathrm{H}_{2} \mathrm{O}$ systems. Enhanced electron stopping is observed in this $\mathrm{D}_{2} \mathrm{O}$ layer and this correlates well with the greater density of $\mathrm{D}_{2} \mathrm{O}$ with respect to $\mathrm{H}_{2} \mathrm{O}$. In particular, the deeper the $\mathrm{D}_{2} \mathrm{O}$ layer is located, the lower the energy of the primary electrons will be at that depth due to scattering in the film. Thus, more electrons will be stopped when transitioning from $\mathrm{H}_{2} \mathrm{O}$ to the deuterated analogue resulting in a more marked peak in the distribution curves in Figure 5 (see samples S1 to S5 in Table 1).

In the range of 300-1000 eV for a fixed structure model of the ASW film, Figure 6 shows the electron trajectories as a function of the incident electron energy. In this model, the buried 
$\mathrm{D}_{2} \mathrm{O}$ layer with a thickness of $\sim 0.2 \mathrm{~nm}$ was deposited on a $60 \mathrm{~nm}$ thick $\mathrm{H}_{2} \mathrm{O}$ layer and capped by another $5.4 \mathrm{~nm}$ thick layer of $\mathrm{H}_{2} \mathrm{O}$. As shown in Figure 5 , the presence of the $\mathrm{D}_{2} \mathrm{O}$ layer is revealed by an enhancement of the electron stopping in the form of a peak. This feature becomes less and less noticeable as the energy of the incident electron beam is increased. In particular, when moving from $400 \mathrm{eV}$ to $500 \mathrm{eV}$, the number of primary electrons that have stopped at the depth of the $\mathrm{D}_{2} \mathrm{O}$ layer decreases by a factor of 17 . This can be readily linked to the fact that for higher electron energies, the difference in density between $\mathrm{D}_{2} \mathrm{O}$ and $\mathrm{H}_{2} \mathrm{O}$ becomes noticeably less important. Interestingly, at higher energies fewer primary electrons thermalise at the depth of the $D_{2} \mathrm{O}$ layer, although those that stopped within the first $5.6 \mathrm{~nm}$ have deposited more energy in the ice with respect to the analogous experiment using lower energy electrons. Also, note that as the energy of the electron beam is increased, the enhancement of the stopping in the $\mathrm{D}_{2} \mathrm{O}$ layer decreases (Figure 6 inset). For instance, beyond $750 \mathrm{eV}$, the electron beam will clearly pass through the whole $44.7 \mathrm{~nm}$ thick compact ASW film reaching the underlying metal substrate while displaying little effect due to the presence of the $\mathrm{D}_{2} \mathrm{O}$ layer.

In an attempt to rationalise the $\mathrm{HD}$ and $\mathrm{D}_{2}$ peak positions after irradiation with $450 \mathrm{eV}$ electrons of $\mathrm{H}_{2} \mathrm{O} / \mathrm{D}_{2} \mathrm{O} / \mathrm{H}_{2} \mathrm{O}$ ASW films in which the $\mathrm{D}_{2} \mathrm{O}$ layer is buried under $\mathrm{H}_{2} \mathrm{O}$ cap layers of various thicknesses (see Figure 3), we have developed a simple Monte Carlo model based on the work of Petrik et al. ${ }^{5}$. We assume that an incoming $450 \mathrm{eV}$ electron creates an 'event' at a certain depth within the ice film where it loses most of its energy ${ }^{65}$. The exact depth of this 'event' is Monte Carlo selected, and could be an ionisation event, or excitation, leading to the production of e.g. a hydrogen atom. If this 'event' takes place at a depth that corresponds to the depth of the $\mathrm{D}_{2} \mathrm{O}$ layer, then a $\mathrm{D}$ atom is formed, otherwise an $\mathrm{H}$ atom is formed. This reactive species is now allowed to travel in any direction, and depending on whether it encounters a $\mathrm{H}_{2} \mathrm{O}$ or $\mathrm{D}_{2} \mathrm{O}$ molecule in the next step, either $\mathrm{H}_{2}, \mathrm{HD}$, or $D_{2}$ is formed. This newly formed hydrogen molecule is now allowed to diffuse in a random manner through a cubic ice matrix in which each monolayer in the ice is represented by one layer in the cube; the $x$ and $y$ dimensions of the cube are 100 molecules each, and the thickness of the slab is $44.5 \mathrm{~nm}$. Periodic boundary conditions in the $x$ and $y$ dimension, i.e. parallel to the vacuum interface, guarantee that no hydrogen is lost. If the hydrogen reaches 
the vacuum interface, however, then the number of random steps from its creation to reaching the vacuum is counted and considered proportional to the arrival time on the mass

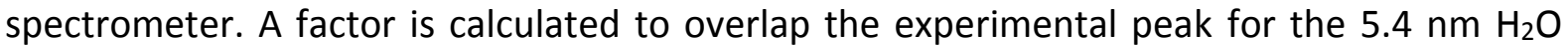
cap coverage experiments with the simulation peak, and the same factor is applied to all other data. A total of two-million electrons are deposited in the ice for each coverage to make up the overall distributions.

Figure 7 shows - compared with the experimental data in panel (a) - the results from these Monte Carlo (MC) simulations in panel (b). It can be seen that the simple model captures the essentials of the experiment rather well, i.e. the longer arrival times of $H D / D_{2}$ for more deeply buried $\mathrm{D}_{2} \mathrm{O}$ layers, the decline in signal intensity, as well as the broadening of the peaks. All three are a direct consequence of the increased diffusion length through the ice with increased $\mathrm{D}_{2} \mathrm{O}$ layer depth. One could argue that our simple model is unable to reproduce the exact kinetics observed in the experimental desorption profiles of $H D$ and $D_{2}$. For instance, in the latter case, see panel (a) in Figure 7, an almost constant contribution to the desorption traces appears to be superimposed to the peaks. This contribution to the recorded signal has little or no equivalent in the simulated curves and could be attributed to isotope exchange during background dosing. However, more experiments are needed to fully assess this problem.

\section{Discussion}

Experiment

As seen in Figures 3 and $\mathbf{4}$, the formation and subsequent desorption of $H_{2}, H D$, and $D_{2}$ is directly and indirectly attributed to the electron-induced dissociation of $\mathrm{H}_{2} \mathrm{O}$ and $\mathrm{D}_{2} \mathrm{O}$ when layered water ices are excited by electrons. The mechanism is rather complex and even though electron irradiation of ASW has been thoroughly investigated some details remain to be clarified ${ }^{44}$. Therefore, for the sake of simplicity, we highlight the three key processes of the mechanism involved:

$$
\begin{array}{ll}
\text { Excitation } & \mathrm{H}_{2} \mathrm{O}+\mathrm{e}^{-} \rightarrow \mathrm{H}_{2} \mathrm{O}^{*} \\
\text { Dissociation } & \mathrm{H}_{2} \mathrm{O}^{*} \rightarrow \mathrm{H}+\mathrm{OH}
\end{array}
$$




$$
\begin{array}{ll} 
& \mathrm{H}_{2} \mathrm{O}^{*}+\mathrm{H}_{2} \mathrm{O} \rightarrow 2 \mathrm{OH}+\mathrm{H}_{2} \\
\mathrm{H}_{2} \text { formation } & \mathrm{H}_{2} \mathrm{O}+\mathrm{H} \rightarrow \mathrm{OH}+\mathrm{H}_{2}
\end{array}
$$

Reaction (1) is electronic excitation of $\mathrm{H}_{2} \mathrm{O}$ by direct interaction with the primary and/or secondary electrons. This electronic excitation can be efficiently transferred to the neighbouring hydrogen-bonded molecules $\left(\mathrm{H}_{2} \mathrm{O}^{*}+\mathrm{H}_{2} \mathrm{O} \rightarrow \mathrm{H}_{2} \mathrm{O}+\mathrm{H}_{2} \mathrm{O}^{*}\right)^{5,53,54,66}$. The excited molecule can then dissociate forming the $\mathrm{OH}, \mathrm{H}$ and $\mathrm{H}_{2}$ [reactions (2a) and (2b)]. The $\mathrm{OH}$ chemistry is incredibly rich and leads to the formation of $\mathrm{HO}_{2}$ which can also contribute to the $\mathrm{H}_{2}$ yield thanks to further reactions ${ }^{67,}{ }^{68}$. Molecular hydrogen is also formed via the abstraction reaction (3). Diffusion of the product $\mathrm{H}_{2}$ through the bulk to the vacuum interface where it desorbs then follows. Moreover, ionisation by removal of a valence electron at electron energies above $\sim 20 \mathrm{eV}$ and core ionisation at high energies, around 540 $e V$, are relevant $\left(\mathrm{H}_{2} \mathrm{O} \rightarrow \mathrm{H}_{2} \mathrm{O}^{+}+\mathrm{e}^{-}\right)$, leading to the formation of charged species such as $\mathrm{H}_{2} \mathrm{O}^{+}$ and $\mathrm{H}_{3} \mathrm{O}^{+} 69,70\left(\mathrm{H}_{2} \mathrm{O}+\mathrm{H}_{2} \mathrm{O}^{+} \rightarrow \mathrm{H}_{3} \mathrm{O}^{+}+\mathrm{OH}\right)$. These cations can recombine with the trapped electrons and result in excited molecules which further contribute to the $\mathrm{H}_{2}$ yield $\left(\mathrm{H}_{3} \mathrm{O}^{+}+\mathrm{e}^{-}\right.$ $\left.\rightarrow \mathrm{H}_{3} \mathrm{O}^{*} \rightarrow \mathrm{H}_{2} \mathrm{O}+\mathrm{H}\right)^{49}$ in addition to simple $\mathrm{H}$ abstraction and dimerisation.

Several previous experimental studies of electron irradiation of ASW have provided unambiguous evidence for $\mathrm{H}_{2}$ forming at the vacuum interface, and not in the bulk of the ice $^{1,2,53}$. If this were to be the dominant mechanism responsible for $\mathrm{H}_{2}$ formation, we would not observe any $\mathrm{D}_{2}$ forming once a sufficiently thick capping layer of $\mathrm{H}_{2} \mathrm{O}$ is deposited on top of the $\mathrm{D}_{2} \mathrm{O}$ layer. This is in clear contrast with our experiments (see Figure $3(\mathbf{c})$ ) which show $D_{2}$ desorbing even when the $D_{2} \mathrm{O}$ layer is located roughly $11 \mathrm{~nm}$ below the vacuum interface in the $\mathrm{H}_{2} \mathrm{O} / \mathrm{D}_{2} \mathrm{O} / \mathrm{H}_{2} \mathrm{O}$ system (see Table 1). Conversely, the traces reported in Figure 3(c)) are consistent with $\mathrm{H}_{2}$ and $\mathrm{D}_{2}$ forming in the bulk, diffusing through the ice and finally desorbing. This interpretation is further strengthened by the simulations in Figure 7. Such apparent contrast with the previously published literature can be easily addressed as due to the different energy of the primary electrons employed in this work. In fact, we could argue that at $100 \mathrm{eV} \mathrm{H}\left(D_{2}\right)$ formation is a surface process following the transportation of the excitation from the bulk to the vacuum interface. Whereas at higher electron energies (above $400 \mathrm{eV}$ ), the main mechanism responsible for the observed desorption traces 
appears to be no longer a surface-specific process, and hence $\mathrm{H}_{2}$ forming in the bulk becomes the dominant phenomenon.

Moreover, the observed differences in the desorption profiles of Figure $\mathbf{4}(\mathbf{b})$ and $\mathbf{4}(\mathbf{c})$ are rather noticeable, as the peak maxima are centred at $35 \mathrm{~s}, 176 \mathrm{~s}$ and $460 \mathrm{~s}$ for $500 \mathrm{eV}, 450$ $\mathrm{eV}$, and $400 \mathrm{eV}$, respectively. However, such large increases between these appearance times are unlikely to be due just to the different kinetics of the primary electrons travelling through the ice itself. In fact, the depth where the electrons do most damage for 400,450 and $500 \mathrm{eV}$ is very close in all the three cases ${ }^{65}$, and hence the different kinetic energy could arguably explain the significant change of hundreds of seconds in the appearance of the desorption peaks as shown in Figure 4. More realistically, the observed trend can be rationalised in three ways:

(i) $D_{2}$ and HD diffusion could be at least partially non-thermal and hence these species would escape the ice more quickly at higher energies, although to the best of our knowledge there is little evidence to support this interpretation;

(ii) As the irradiation proceeds, the electrons will continuously reduce the distance between the vacuum interface and the buried $\mathrm{D}_{2} \mathrm{O}$ layer via sputtering (read as desorption) of the upper $\mathrm{H}_{2} \mathrm{O}$ layers. This means that if $500 \mathrm{eV}$ electrons are associated with a larger cross section for $\mathrm{H}_{2} \mathrm{O}$ electron-promoted desorption than $400 \mathrm{eV}$ electrons, the ice would be eroded more efficiently at higher energies;

(iii) Alternatively, given the fact that $500 \mathrm{eV}$ electrons are energetically close to the ionisation threshold (ca. $538.1 \mathrm{eV}$ ) of the core $\mathrm{O} 1 \mathrm{~s}$ orbital in condensed water, a different excitation mechanism might be involved and/or become more relevant.

\section{Simulation}

We report in Figure 8 the non-linear relationship between $H D / D_{2}$ production peak times and the depth of the $\mathrm{D}_{2} \mathrm{O}$ layer as displayed in panels (a) and (b) of Figure 7. The resulting two 
data sets are comparable but the $M C$ data shows faster diffusion toward the vacuum interface and slower diffusion at depth. The simple model used in the MC simulations is capable of qualitatively describing the experimental trend and captures the dominant process responsible for it, but these simulations fail in reproducing quantitatively the exact behaviour that we observe. This means that HD diffusion is the key step that "shapes" the trend, but that there are other physicochemical processes involved that (a) delay the appearance of $\mathrm{HD}$ for thin $\mathrm{H}_{2} \mathrm{O}$ caps, and (b) accelerate the release of $\mathrm{HD}$ for thicker $\mathrm{H}_{2} \mathrm{O}$ caps. The nature of these processes is unclear and will be subject further investigation in the development of more sophisticated simulations.

Having established the scaling factor to convert from the number of random steps to appearance time as we reported in the results section, we also attempted to include the effects of erosion caused by the electrons as the electron beam is continuously irradiating a point on the ice film during a typical experiment. Each electron was assigned a separate randomly-selected delay time (between 0 and 600 seconds) and the degree of ice erosion that will have occurred before a particular electron penetrates the ice is calculated. This calculation is based on the known electron flux and on the interpolated erosion rates previously measured in this laboratory $\left(0.085 \mathrm{ML} \mathrm{s}^{-1}\right.$ for $400 \mathrm{eV}$ electrons, $0.102 \mathrm{ML} \mathrm{s}^{-1}$ for $450 \mathrm{eV}$ electrons, and $0.12 \mathrm{ML} \mathrm{s}^{-1}$ for $500 \mathrm{eV}$ electrons ${ }^{1,46-50}$ ). The HD and $\mathrm{D}_{2}$ appearance times are now a function of the (delay) time within the 10 minutes irradiation interval at which a particular electron initially interacted with the ice (due to the greater degree of erosion at later times), and of the diffusion of the product hydrogen molecule to the surface. While the penetration depth of the $500 \mathrm{eV}$ electrons is deeper than for the $450 \mathrm{eV}$ electrons, which in turn is deeper than for the $400 \mathrm{eV}$ electrons, the thickness of the effective $\mathrm{H}_{2} \mathrm{O}$ cap layer is reduced due to erosion. This effect consequently causes the HD and $D_{2}$ peaks due to the $500 \mathrm{eV}$ electrons to appear at earlier times as compared to the $450 \mathrm{eV}$ electrons (which again are earlier than the $400 \mathrm{eV}$ electrons, which cause the least erosion). The simulations do not quantitatively reproduce the effect seen in the experiments, but at least mimic the (counterintuitive) trend observed, namely that the faster and deeper penetrating electrons are responsible for the earlier $H D / D_{2}$ peaks. 


\section{Astrophysical Implications}

Non-thermal processing is a key mechanism for increasing chemical complexity in astrophysical ices. This work also suggests that it has an additional role in promoting the formation of the simplest of all molecules;

(1) Radiolytic electron-induced chemistry, promoted by ionising photons and cosmic rays, is an efficient mechanism for returning $\mathrm{H}_{2}$ (and its isotopologues) to the gas phase from $\mathrm{H}_{2} \mathrm{O}$ ices. Whether this is true for other molecular solids commonly found in the interstellar medium will necessarily require further work. Such a process will necessarily impact on the gas phase chemistry of evolving objects and this warrants further investigation especially its potential impact on $\mathrm{H}_{2}$ isotopologue abundances.

In addition, this work may point to a simple approach to understanding physics and chemistry involving icy solids;

(2) The experiments and simulations reported herein point to a relatively simple tworegion model for chemistry in icy grains. First, there is the selvedge, or near-surface region, from which desorbing species can escape with minimal inelastic scattering from the atoms and molecules in this region. Diffusion plays little or no role in this region. Below selvedge, we have the bulk solid in which diffusion is the key physical process. Direct desorption, even moderated by inelastic scattering is impossible and volatiles produced by electronic excitation and subsequent reactions must diffuse through the bulk medium to escape. Further experiments at lower temperature and additional simulations are required to more fully develop our selvedge-bulk model.

\section{Conclusions}

EIC in layered ASW systems comprising of $\mathrm{H}_{2} \mathrm{O}$ and $\mathrm{D}_{2} \mathrm{O}$ leads to the production of hydrogen isotopologues $\left(H_{2}, H D\right.$, and $\left.D_{2}\right)$ which we have investigated as a function of electron energy 
and penetration depth. Maxima in the production rate of $H D$ and $D_{2}$ and a minimum in that of $\mathrm{H}_{2}$ are observed to move to longer times as the $\mathrm{D}_{2} \mathrm{O}$ probe layer is buried further within the ASW film. In addition, these features broaden with $\mathrm{D}_{2} \mathrm{O}$ depth as a consequence of the diffusion of the primary atomic and secondary molecular species. A similar trend is observed in these peaks when changing the electron energy, from $400 \mathrm{eV}$ to $450 \mathrm{eV}$, up to $500 \mathrm{eV}$, for an ASW film with a $\mathrm{D}_{2} \mathrm{O}$ inter-layer at a fixed depth.

Simulations using the CASINO code indicate that the electrons used can penetrate to the $\mathrm{D}_{2} \mathrm{O}$ inter-layer and represent a preliminary view of the physics occurring in this system. More detailed Monte Carlo modelling of hydrogen isotopologue production shows the longer arrival times of $H D / D_{2}$ for more deeply buried $\mathrm{D}_{2} \mathrm{O}$ layers. The decline in the signal intensity, as well as the broadening of the deepest peak production as a result of the increased diffusion length through the ice are consistent with the experimental observations supporting the idea that the detected molecular species are formed within the bulk.

\section{Acknowledgments}

This work was financially supported by the Egyptian Science \& Technology Development Fund (STDF; Project ID:6119). We thank all the team at the STDF. DM clarifies that his contribution to this work has been done as a private venture and not in the author's capacity as an affiliate of the Jet Propulsion Laboratory, California Institute of Technology.

\section{References}

1. N. G. Petrik and G. A. Kimmel, Phys. Rev. Lett., 2003, 90, 166102.

2. N. G. Petrik and G. A. Kimmel, J. Chem. Phys., 2004, 121, 3736.

3. A. Yabushita, T. Hama and M. Kawasaki, J. Photoch. Photobio. C-Photochemistry Reviews, 2013, 16, 46.

4. J. D. Thrower, M. P. Collings, F. J. M. Rutten and M. R. S. McCoustra, Chem. Phys. Lett., 2011, 505, 106.

5. N. G. Petrik, R. J. Monckton, S. P. K. Koehler and G. A. Kimmel, J. Chem. Phys., 2014, 140, 204710. 
6. C. J. Bennett, T. Hama, Y. S. Kim, M. Kawasaki and R. I. Kaiser, Astrophys. J., 2011, 727, 27.

7. R. E. Johnson and T. I. Quickenden, J. Geophys. Res - Planet, 1997, 102, 10985-10996.

8. T. E. Madey, R. E. Johnson and T. M. Orlando, Surf. Sci., 2002, 500, 838.

9. P. Jenniskens and D. F. Blake, Science, 1994, 265, 753.

10. R. Smoluchowski, Science, 1978, 201, 809.

11. A. G. G. M. Tielens and D. C. B. Whittet, lau Symp, 1997, 45.

12. E. Herbst and E. F. van Dishoeck, Annu. Rev. Astron. Astrophys., 2009, 47, 427.

13. A. C. A. Boogert, K. M. Pontoppidan, C. Knez, F. Lahuis, J. Kessler-Silacci, E. F. van Dishoeck, G. A. Blake, J. C. Augereau, S. E. Bisschop, S. Bottinelli, T. Y. Brooke, J. Brown, A. Crapsi, N. J. Evans, H. J. Fraser, V. Geers, T. L. Huard, J. K. Jorgensen, K. I. Oberg, L. E. Allen, P. M. Harvey, D. W. Koerner, L. G. Mundy, D. L. Padgett, A. I. Sargent and K. R. Stapelfeldt, Astrophys. J., 2008, 678, 985.

14. J. E. Chiar, A. J. Adamson, T. H. Kerr and D. C. B. Whittet, Astrophys. J., 1995, 455, 234.

15. E. L. Gibb, D. C. B. Whittet, W. A. Schutte, A. C. A. Boogert, J. E. Chiar, P. Ehrenfreund, P. A. Gerakines, J. V. Keane, A. G. G. M. Tielens, E. F. van Dishoeck and O. Kerkhof, Astron. Astrophys., 2000, 536, 347.

16. E. L. Gibb, D. C. B. Whittet, A. C. A. Boogert and A. G. G. M. Tielens, Astrophys. J. Suppl. Ser., 2004, 151, 35.

17. K. M. Pontoppidan, Astron. Astrophys., 2006, 453, L47.

18. K. M. Pontoppidan, H. J. Fraser, E. Dartois, W. F. Thi, E. F. van Dishoeck, A. C. A. Boogert, L. d'Hendecourt, A. G. G. M. Tielens and S. E. Bisschop, Astron. Astrophys., 2003, 408, 981.

19. M. Tanaka, T. Nagata, S. Sato and T. Yamamoto, Astrophys.l J., 1994, 430, 779.

20. A. G. G. M. Tielens, A. T. Tokunaga, T. R. Geballe and F. Baas, Astrophys. J., 1991, 381, 181.

21. G. Zasowski, F. Kemper, D. M. Watson, E. Furlan, C. J. Bohac, C. Hull and J. D. Green, Astrophys. J., 2009, 694, 459.

22. E. F. van Dishoeck, E. Herbst and D. A. Neufeld, Chem. Rev., 2013, 113, 9043.

23. A. G. G. M. Tielens, Astron. Astrophys., 1983, 119, 177.

24. S. B. Charnley, A. G. G. M. Tielens and S. D. Rodgers, Astrophys. J., 1997, 482, L203. 
25. H. M. Butner, S. B. Charnley, C. Ceccarelli, S. D. Rodgers, J. R. Pardo, B. Parise, J. Cernicharo and G. R. Davis, Astrophys. J., 2007, 659, L137.

26. C. Vastel, C. Ceccarelli, E. Caux, A. Coutens, J. Cernicharo, S. Bottinelli, K. Demyk, A. Faure, L. Wiesenfeld, Y. Scribano, A. Bacmann, P. Hily-Blant, S. Maret, A. Walters, E. A. Bergin, G. A. Blake, A. Castets, N. Crimier, C. Dominik, P. Encrenaz, M. Gerin, P. Hennebelle, C. Kahane, A. Klotz, G. Melnick, L. Pagani, B. Parise, P. Schilke, V. Wakelam, A. Baudry, T. Bell, M. Benedettini, A. Boogert, S. Cabrit, P. Caselli, C. Codella, C. Comito, E. Falgarone, A. Fuente, P. F. Goldsmith, F. Helmich, T. Henning, E. Herbst, T. Jacq, M. Kama, W. Langer, B. Lefloch, D. Lis, S. Lord, A. Lorenzani, D. Neufeld, B. Nisini, S. Pacheco, J. Pearson, T. Phillips, M. Salez, P. Saraceno, K. Schuster, X. Tielens, F. van der Tak, M. H. D. van der Wiel, S. Viti, F. Wyrowski, H. Yorke, P. Cais, J. M. Krieg, M. Olberg and L. Ravera, Astron. Astrophys., 2010, 521, L31.

27. L. E. Kristensen, L. Amiaud, J. H. Fillion, F. Dulieu and J. L. Lemaire, Astron. Astrophys., 2011, 527, A44.

28. C. Ceccarelli, Planet. Space Sci., 2002, 50, 1267.

29. E. Roueff and M. Gerin, Space. Sci. Rev., 2003, 106, 61.

30. J. K. Jorgensen and E. F. van Dishoeck, Astrophys. J. Lett., 2010, 725, L172.

31. F. C. Liu, B. Parise, L. Kristensen, R. Visser, E. F. van Dishoeck and R. Gusten, Astron. Astrophys., 2011, 527, A19.

32. A. Coutens, C. Vastel, E. Caux, C. Ceccarelli, S. Bottinelli, L. Wiesenfeld, A. Faure, Y. Scribano and C. Kahane, Astron. Astrophys., 2012, 539, A132.

33. A. Coutens, C. Vastel, S. Cabrit, C. Codella, L. E. Kristensen, C. Ceccarelli, E. F. van Dishoeck, A. C. A. Boogert, S. Bottinelli, A. Castets, E. Caux, C. Comito, K. Demyk, F. Herpin, B. Lefloch, C. McCoey, J. C. Mottram, B. Parise, V. Taquet, F. F. S. van der Tak, R. Visser and U. A. Yildiz, Astron. Astrophys., 2013, 560, A39.

34. M. V. Persson, J. K. Jorgensen and E. F. van Dishoeck, Astron. Astrophys., 2013, 549, L3.

35. V. Taquet, A. Lopez-Sepulcre, C. Ceccarelli, R. Neri, C. Kahane, A. Coutens and C. Vastel, Astrophys. J. Lett., 2013, 768, L29.

36. M. V. Persson, J. K. Jorgensen, E. F. van Dishoeck and D. Harsono, Astron. Astrophys., 2014, 563, A74. 
37. A. Coutens, J. K. Jorgensen, M. V. Persson, E. F. van Dishoeck, C. Vastel and V. Taquet, Astrophys. J. Lett., 2014, 792, L5.

38. A. Faure, M. Faure, P. Theule, E. Quirico and B. Schmitt, Astron. Astrophys., 2015, 584, A98.

39. K. Furuya, E. F. van Dishoeck and Y. Aikawa, Astron. Astrophys., 2016, 586, A127.

40. A. Punanova, P. Caselli, A. Pon, A. Belloche and P. Andre, Astron. Astrophys., 2016, 587, A118.

41. L. I. Cleeves, E. A. Bergin, C. M. O. Alexander, F. J. Du, D. Graninger, K. I. Oberg and T. J. Harries, Astrophys. J., 2016, 819, 13.

42. F. Fontani, G. Busquet, A. Palau, P. Caselli, A. Sanchez-Monge, J. C. Tan and M. Audard, Astron. Astrophys., 2015, 575, A87.

43. B. Boudaiffa, P. Cloutier, D. Hunting, M. A. Huels and L. Sanche, Science, 2000, 287, 1658.

44. B. C. Garrett, D. A. Dixon, D. M. Camaioni, D. M. Chipman, M. A. Johnson, C. D. Jonah, G. A. Kimmel, J. H. Miller, T. N. Rescigno, P. J. Rossky, S. S. Xantheas, S. D. Colson, A. H. Laufer, D. Ray, P. F. Barbara, D. M. Bartels, K. H. Becker, H. Bowen, S. E. Bradforth, I. Carmichael, J. V. Coe, L. R. Corrales, J. P. Cowin, M. Dupuis, K. B. Eisenthal, J. A. Franz, M. S. Gutowski, K. D. Jordan, B. D. Kay, J. A. LaVerne, S. V. Lymar, T. E. Madey, C. W. McCurdy, D. Meisel, S. Mukamel, A. R. Nilsson, T. M. Orlando, N. G. Petrik, S. M.

Pimblott, J. R. Rustad, G. K. Schenter, S. J. Singer, A. Tokmakoff, L. S. Wang, C. Wittig and T. S. Zwier, Chem. Rev., 2005, 105, 355.

45. A. Fujishima, X. T. Zhang and D. A. Tryk, Surf. Sci. Rep., 2008, 63, 515.

46. P. Rowntree, L. Parenteau and L. Sanche, J. Chem. Phys., 1991, 94, 8570.

47. G. A. Kimmel and T. M. Orlando, Phys. Rev. Lett., 1995, 75, 2606.

48. G. A. Kimmel, T. M. Orlando, P. Cloutier and L. Sanche, J. Phys. Chem. B, 1997, 101, 6301.

49. N. G. Petrik and G. A. Kimmel, J. Chem. Phys., 2004, 121, 3727.

50. J. D. Thrower, A. G. M. Abdulgalil, M. P. Collings, M. R. S. McCoustra, D. J. Burke, W. A. Brown, A. Dawes, P. J. Holtom, P. Kendall, N. J. Mason, F. Jamme, H. J. Fraser and F. J. M. Rutten, J. Vac. Sci. Technol. A, 2010, 28, 799.

51. G. A. Kimmel and T. M. Orlando, Phys. Rev. Lett., 1996, 77, 3983. 
52. T. M. Orlando and G. A. Kimmel, Surf. Sci., 1997, 390, 79.

53. N. G. Petrik and G. A. Kimmel, J. Chem. Phys., 2005, 123, 054702.

54. N. G. Petrik, R. J. Monckton, S. P. K. Koehler and G. A. Kimmel, J. Phys. Chem. C, 2014, 118, 27483.

55. P. Hovington, D. Drouin, R. Gauvin, D. C. Joy and N. Evans, Scanning, 1997, 19, 29.

56. D. J. Oakes, M. R. S. Mccoustra and M. A. Chesters, Faraday Discuss., 1993, 96, 325.

57. J. D. Thrower, M. P. Collings, F. J. M. Rutten and M. R. S. McCoustra, J. Chem. Phys., $2009,131,244711$.

58. J. D. Thrower, M. P. Collings, F. J. M. Rutten and M. R. S. McCoustra, Mon. Not. R. Astron. Soc., 2009, 394, 1510.

59. A. G. M. Abdulgalil, D. Marchione, A. Rosu-Finsen, M. P. Collings and M. R. S. McCoustra, J Vac Sci Technol A, 2012, 30, 041505.

60. M. S. Westley, G. A. Baratta and R. A. Baragiola, J. Chem. Phys., 1998, 108, 3321.

61. D. E. Brown, S. M. George, C. Huang, E. K. L. Wong, K. B. Rider, R. S. Smith and B. D. Kay, J. Phys. Chem., 1996, 100, 4988.

62. R. L. Summers, Empirical observations on the sensitivity of hot cathode ionization type vacuum gages, 1969.

63. M. P. Seah and W. A. Dench, Surf. Interface Anal. , 1979, 1, 2.

64. D. E. Brown and S. M. George, J. Phys. Chem., 1996, 100, 15460.

65. S. M. Pimblott, J. A. LaVerne and A. Mozumder, J. Phys. Chem. , 1996, 100, 8595.

66. D. Marchione and M. R. S. McCoustra, Phys. Chem. Chem. Phys., 2016, 18, 4026.

67. N. G. Petrik, A. G. Kavetsky and G. A. Kimmel, J. Phys. Chem. B, 2006, 110, 2723.

68. N. G. Petrik, A. G. Kavetsky and G. A. Kimmel, J. Chem. Phys., 2006, 125, 124702.

69. B. Winter, E. F. Aziz, U. Hergenhahn, M. Faubel and I. V. Hertel, J. Chem. Phys., 2007, 126, 124504.

70. S. Thurmer, M. Oncak, N. Ottosson, R. Seidel, U. Hergenhahn, S. E. Bradforth, P. Slavicek and B. Winter, Nat Chem, 2013, 5, 590.

71. D. Drouin, A. R. Couture, D. Joly, X. Tastet, V. Aimez and R. Gauvin, Scanning, 2007, 29, 92. 
Figures 
Figure 1: Schematic diagram of the layered ASW thin film on the stainless steel substrate.

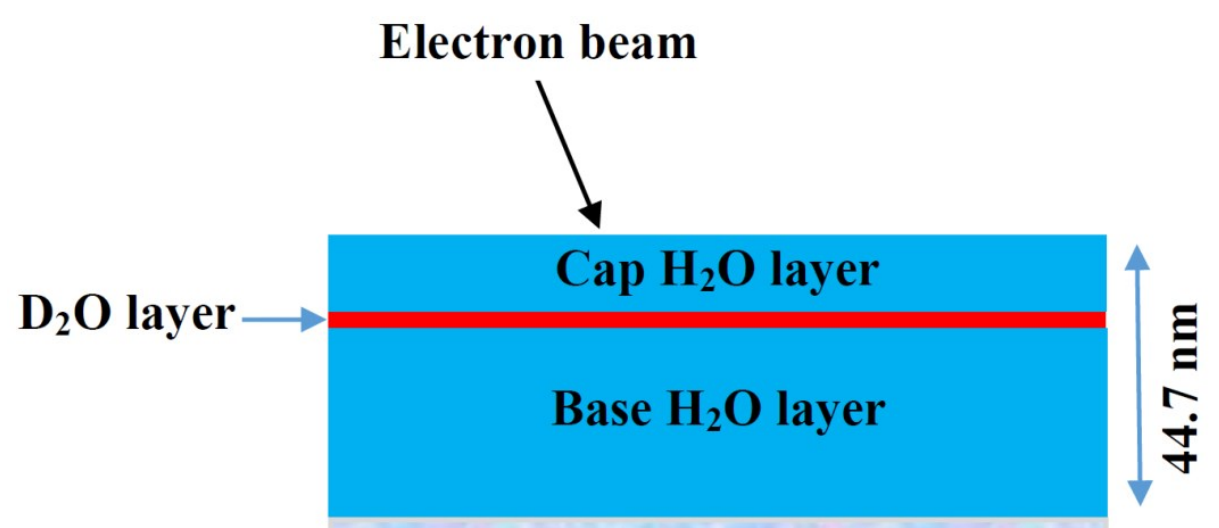

Stainless steel disc $(10 \mathrm{~mm}$

diameter, 2 mm thickness) 
Figure 2: The calculated fraction of the relative electron flux $\left(1 / \mathrm{I}_{0}\right)$ versus depth for electrons incident on the ASW at different energies in the 200 to $1000 \mathrm{eV}$ range.

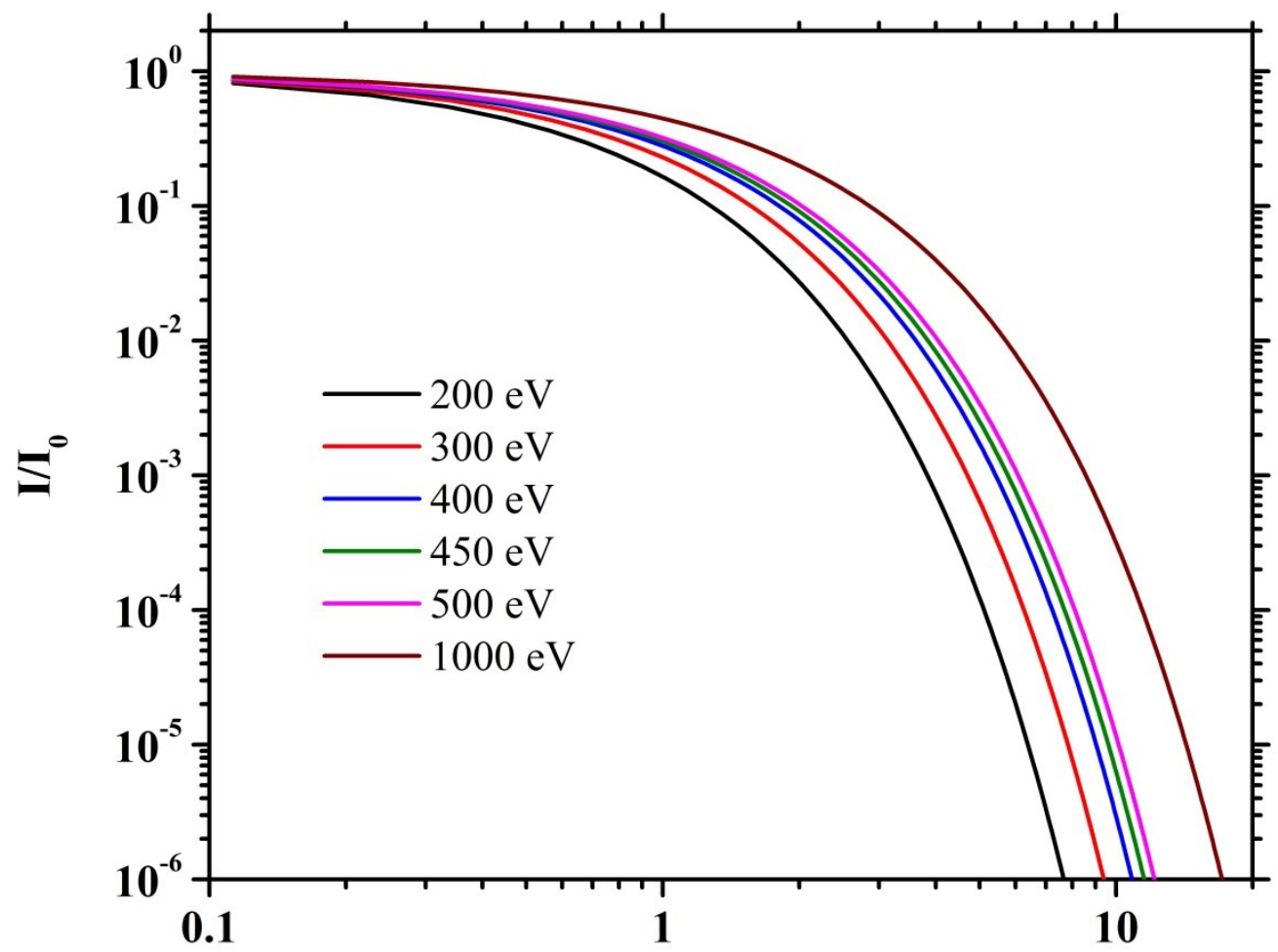

Electron penetration depth (nm) 
Figure 3: The depth dependence of the yields of (a) $H_{2}$, (b) $H D$, and (c) $D_{2}$ molecules produced from thin films of ASW layered as $\mathrm{H}_{2} \mathrm{O} / \mathrm{D}_{2} \mathrm{O} / \mathrm{H}_{2} \mathrm{O}$ for samples; $\mathrm{S} 1$ (black), $\mathrm{S} 2$ (red), S3 (blue), S4 (green) and S5 (magenta) (see Table 1). All samples were irradiated with 450 $\mathrm{eV}$ electrons. The experiment started a time zero, but the electron gun started emitting electrons at $180 \mathrm{sec}$. For clarity, data from S2 to S5 in panel (a) were shifted vertically by $5.0 \times 10^{-11}, 7.0 \times 10^{-11}, 1.5 \times 10^{-10}$, and $1.7 \times 10^{-10}$ molecules $\mathrm{cm}^{-2} \mathrm{~s}^{-1}$, respectively. The dashed vertical lines refer to the time of the reduction in $\mathrm{H}_{2}$ signal and peak production of $\mathrm{HD}$ and $D_{2}$.

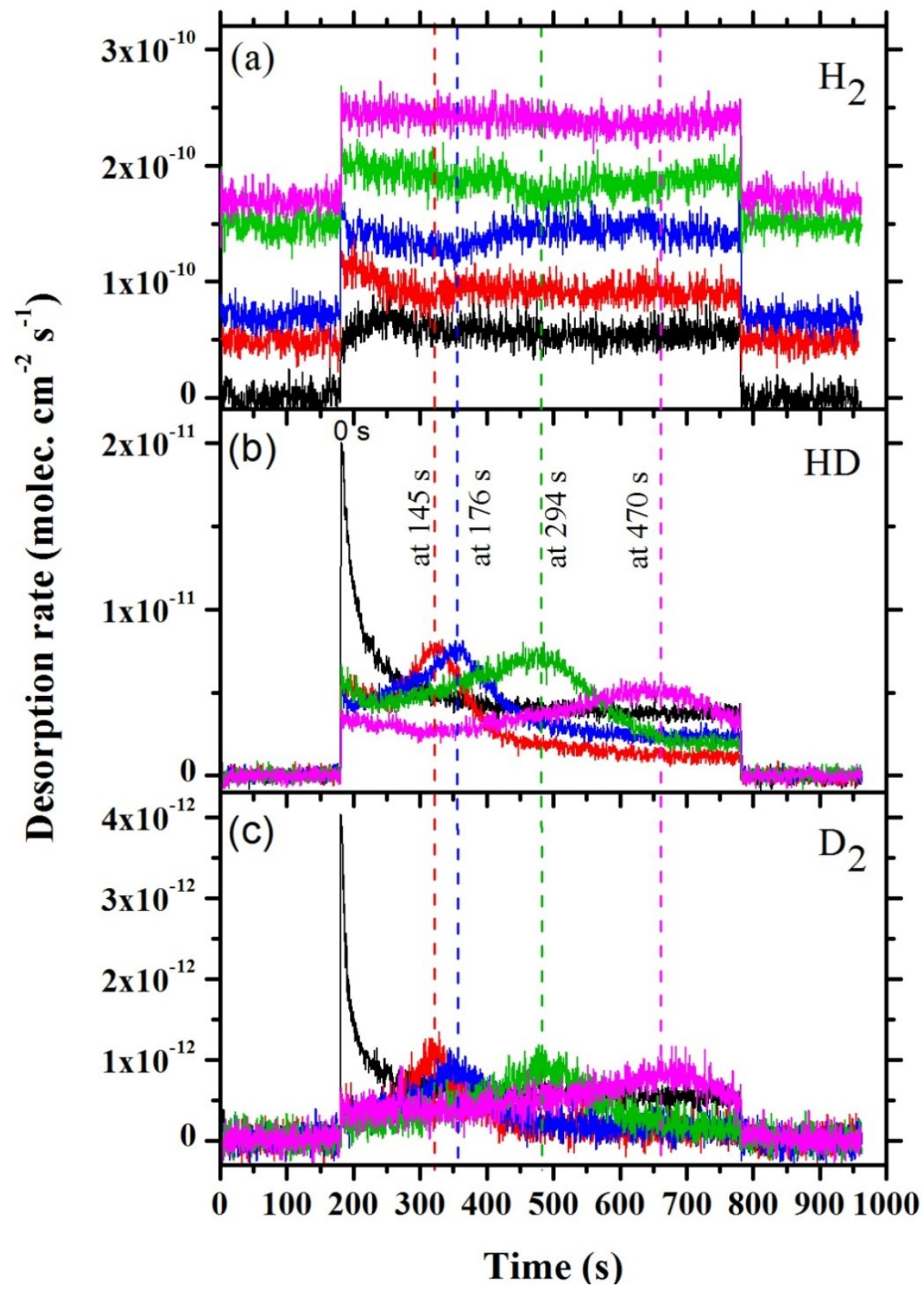


Figure 4: The energy dependence of the yields of (a) $H_{2}$, (b) $H D$, and (c) $D_{2}$ molecules produced from thin films of ice ASW layered as $\mathrm{H}_{2} \mathrm{O} / \mathrm{D}_{2} \mathrm{O} / \mathrm{H}_{2} \mathrm{O}$ for samples; $\mathrm{S} 6$ (black), S3 (red), and S7 (blue) irradiated with 400, 450, and 500 eV electrons, respectively. The experiment started a time zero, but the electron gun started emitting electrons at $180 \mathrm{sec}$. For clarity, the data for samples S3 and S7 in panel $(a)$ were shifted vertically by $7.1 \times 10^{-12}$ and $1.2 \times 10^{-10}$ molecules $\mathrm{cm}^{-2} \mathrm{~s}^{-1}$, respectively. The dashed vertical lines refer to the time of the reduction in $\mathrm{H}_{2}$ signal and peak production of $H D$ and $\mathrm{D}_{2}$ according to the colour.

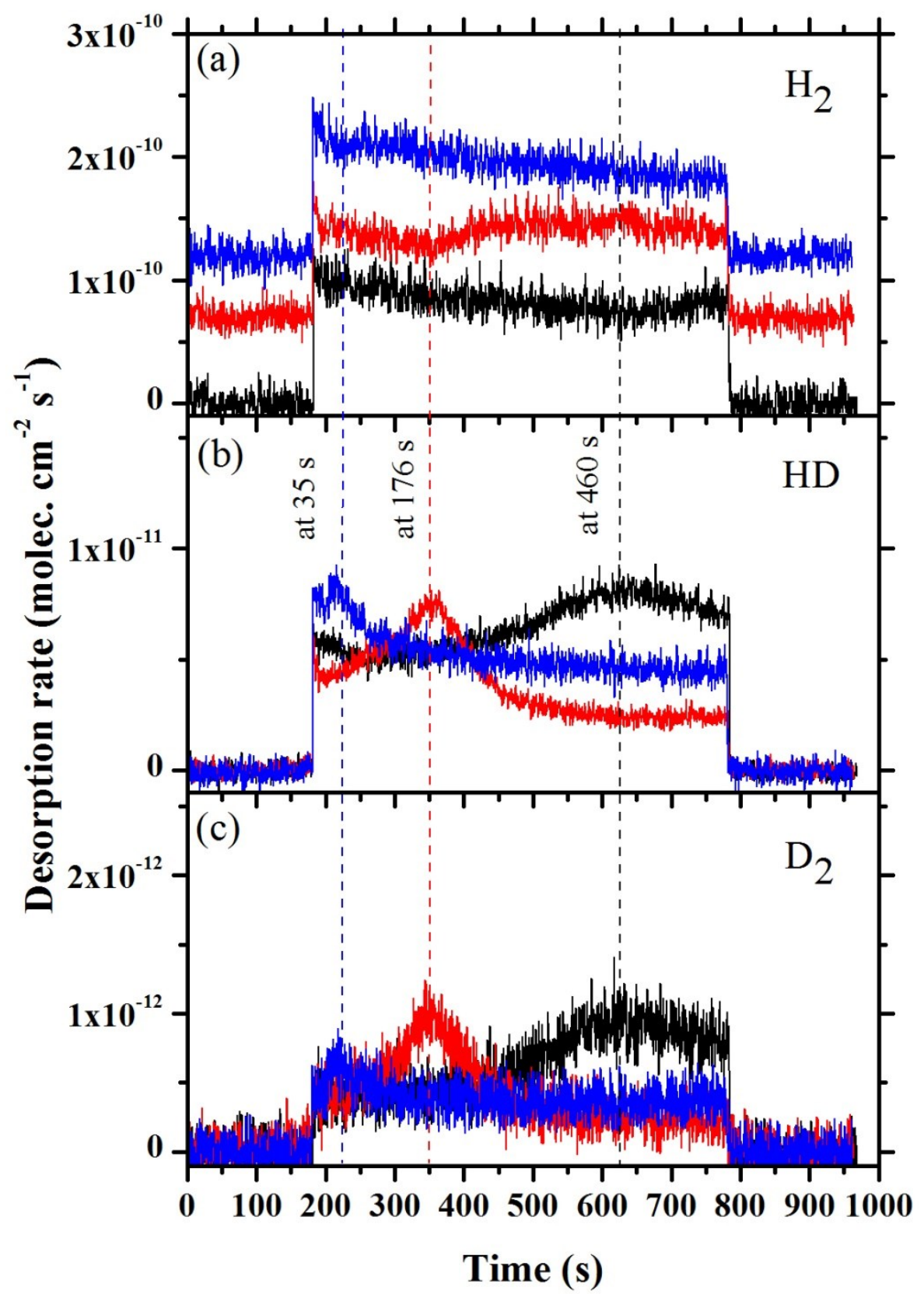


Figure 5: Calculation of the electron trajectories as a function of the penetration depth using the CASINO code and layered icy models of ASW at $450 \mathrm{eV}$. In each model, the $\mathrm{D}_{2} \mathrm{O}$ layer $(0.2 \mathrm{~nm})$ was sandwiched between the base and cap layers arranged at the same conditions of samples S1 (black), S2 (red), S3 (blue), S4 (green) and S5 (magenta) in Table 1. The curves are normalized by the CASINO software by the number of primary electrons simulated ${ }^{71}$. The final four samples were vertically shifted by $2.3 \times 10^{-4}, 4.3 \times 10^{-4}, 8.0 \times 10^{-4}$ and $1.1 \times 10^{-3}$, respectively.

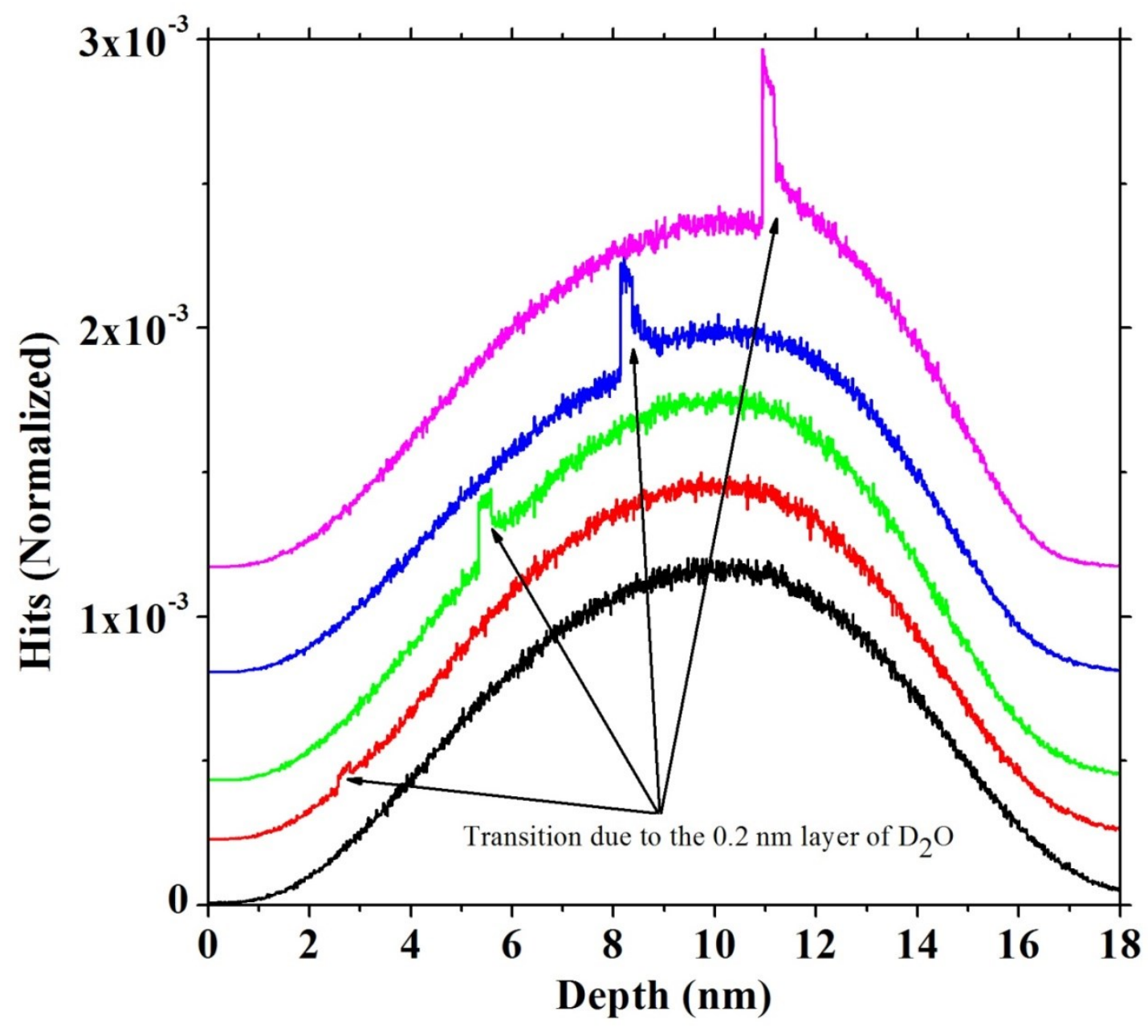


Figure 6: Calculation of the distribution of the primary electrons as a function in the electron energy in the range from 300 to $1000 \mathrm{eV}$ using the CASINO code and model layered ASW film with $\mathrm{H}_{2} \mathrm{O} / \mathrm{D}_{2} \mathrm{O} / \mathrm{H}_{2} \mathrm{O}$ thicknesses of $60 / 0.2 / 5.4 \mathrm{~nm}$, respectively.

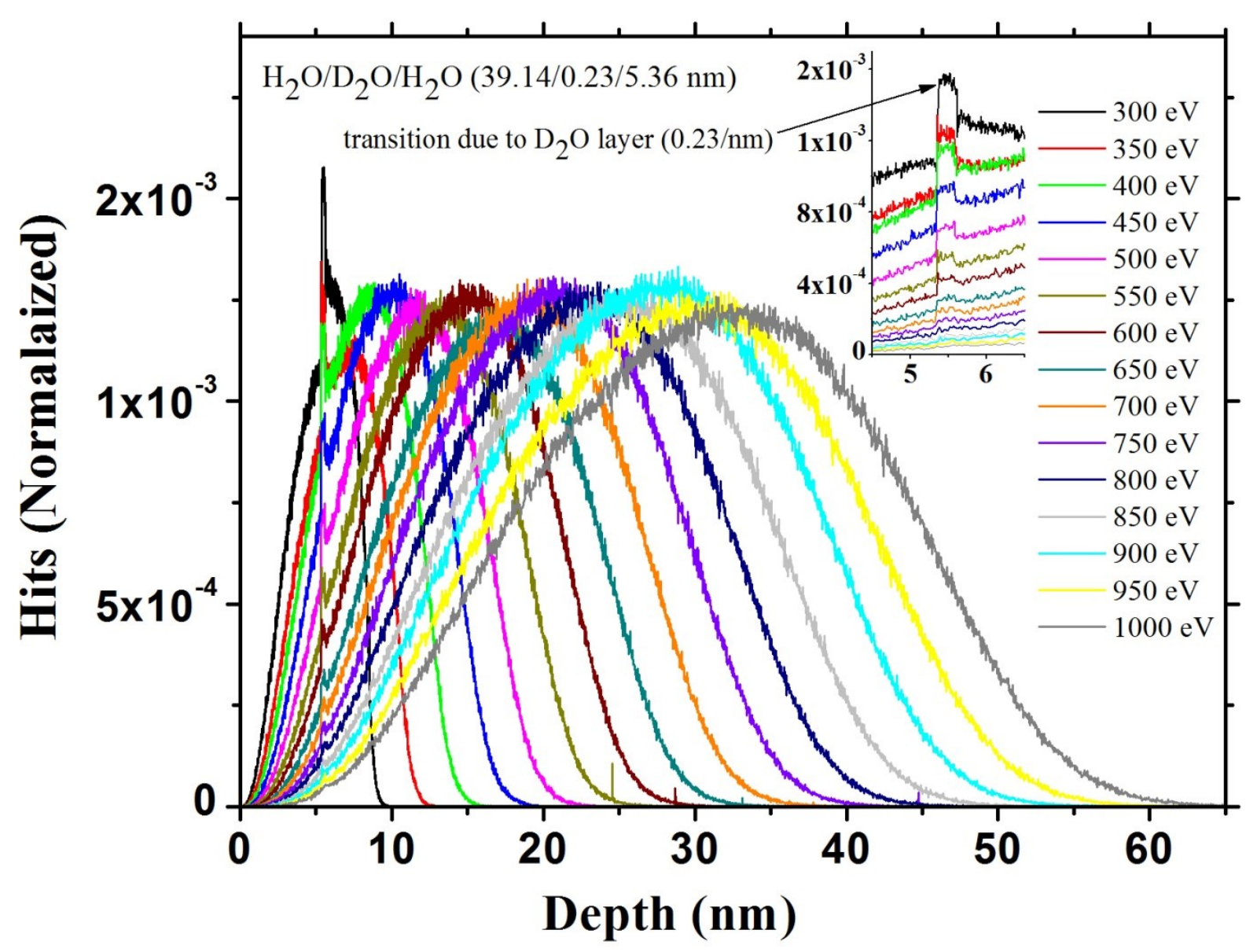


Figure 7: The depth dependence of the yields of HD: (a) produced from thin films of ASW layered as $\mathrm{H}_{2} \mathrm{O} / \mathrm{D}_{2} \mathrm{O} / \mathrm{H}_{2} \mathrm{O}$ for samples when the experiment started a time zero but the electron gun started emitting electrons at 180 sec.; S1 (black), S2 (red), S3 (blue), S4 (green) and S5 (magenta) (Table 1) and (b) the results from MC simulation at the same conditions of samples from S1 to S5. All films were exposed to $450 \mathrm{eV}$ electrons.

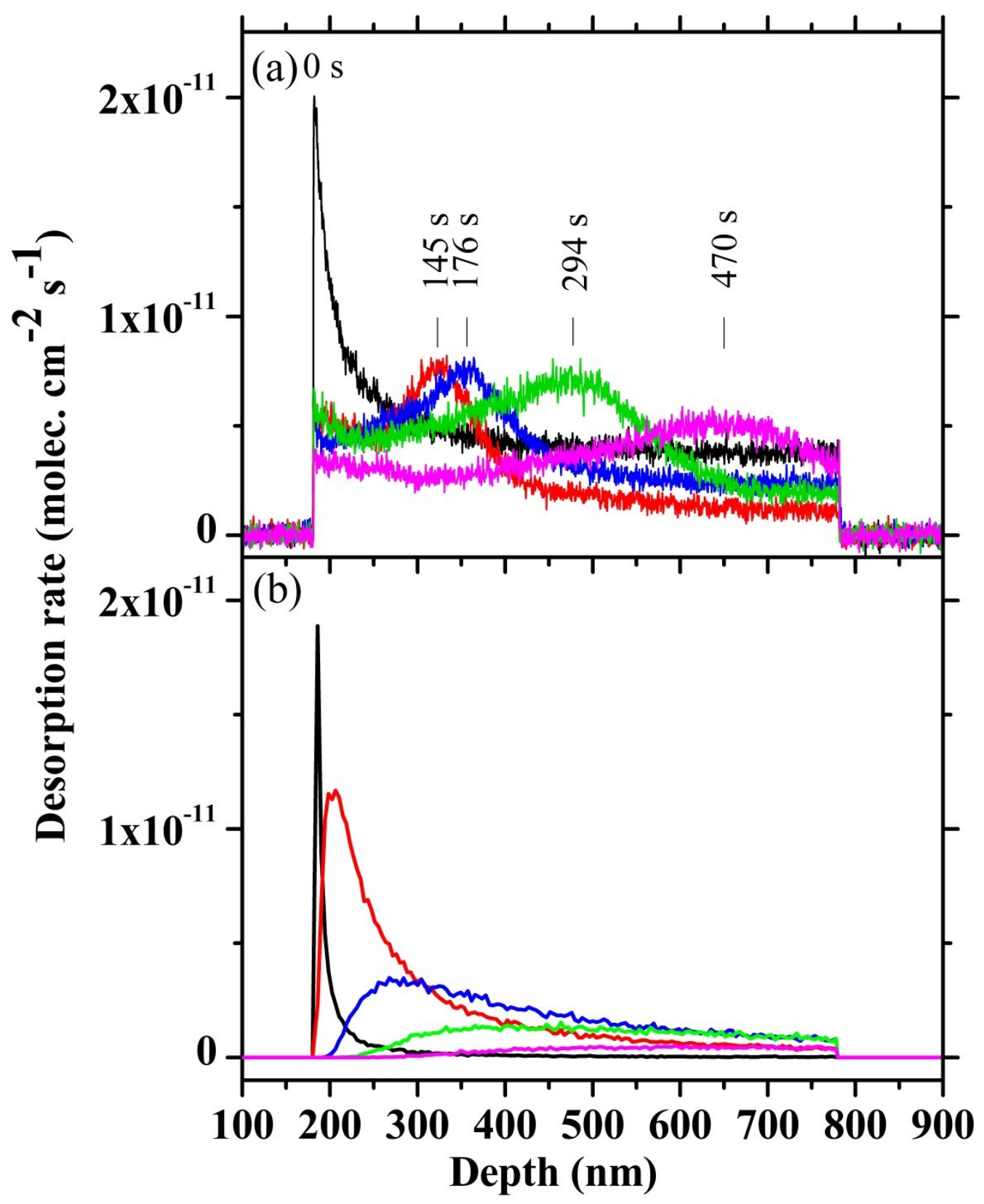


Figure 8: Appearance time of desorption peaks for $H D$ and $D_{2}$ and $M C$ simulation production/desorption of HD peak maxima as a function of the depth of the D2O layer in thin $\mathrm{H}_{2} \mathrm{O} / \mathrm{D}_{2} \mathrm{O} / \mathrm{H}_{2} \mathrm{O}$ ices when irradiated at $450 \mathrm{eV}$ electrons.

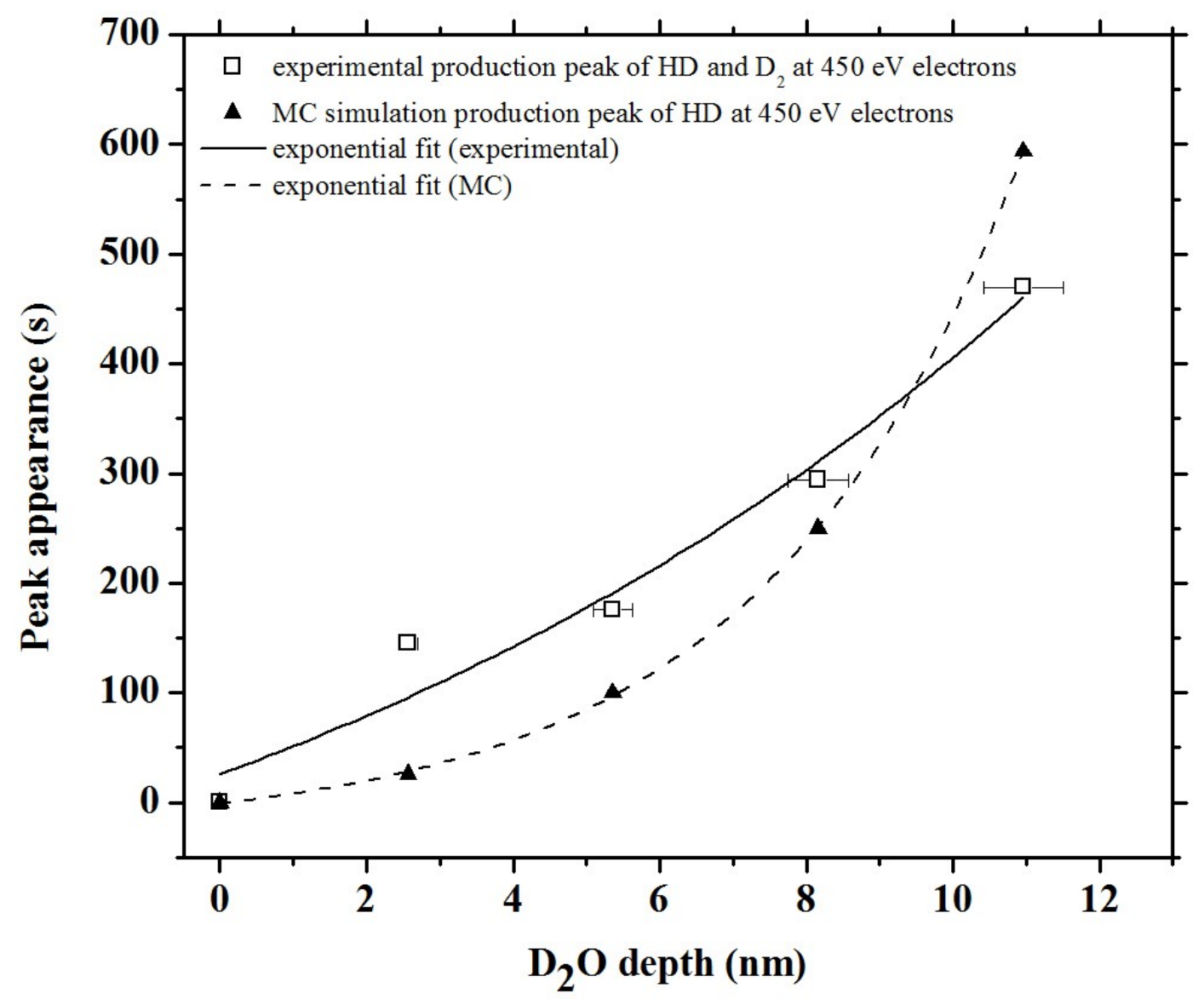


Tables 
Table 1: Layered ASW films of total thickness $44.7 \mathrm{~nm}$ (corresponding to a total exposure of $363 \mathrm{~L}$ ) deposited in UHV on a stainless steel substrate at $108 \mathrm{~K}$. "Samples from S1 to S5 having different layer thicknesses were used to investigate depth-dependence of the $\mathrm{H}_{2}, \mathrm{HD}$ and $D_{2}$ desorption when irradiated with $450 \mathrm{eV}$ electrons for 10 minutes. Samples S6, S3 and S7 having the same layer thicknesses were used in the studies of the energy dependence of the $\mathrm{H}_{2}, \mathrm{HD}$ and $\mathrm{D}_{2}$ desorption when they were irradiated with 400, 450, $500 \mathrm{eV}$ electrons.

\begin{tabular}{|c|c|c|c|}
\hline Sample* & Base $\mathrm{H}_{2} \mathrm{O}$ layer & $\mathbf{D}_{2} \mathrm{O}$ layer & Cap $\mathrm{H}_{2} \mathrm{O}$ layer \\
\hline \multirow{2}{*}{$\mathrm{S} 1$} & $44.5 \pm 2.2 \mathrm{~nm}$ & $0.2 \pm 0.01 \mathrm{~nm}$ & $0.0 \mathrm{~nm}$ \\
& $(361 \pm 18 \mathrm{~L})$ & $(1.8 \pm 0.1 \mathrm{~L})$ & $(0.0 \mathrm{~L})$ \\
\hline \multirow{2}{*}{ S2 } & $41.9 \pm 2.1 \mathrm{~nm}$ & $0.2 \pm 0.01 \mathrm{~nm}$ & $2.6 \pm 0.1 \mathrm{~nm}$ \\
& $(340 \pm 17 \mathrm{~L})$ & $(1.8 \pm 0.1 \mathrm{~L})$ & $(20 \pm 1.0 \mathrm{~L})$ \\
\hline \multirow{2}{*}{ S3 } & $39.1 \pm 2.0 \mathrm{~nm}$ & $0.2 \pm 0.01 \mathrm{~nm}$ & $5.4 \pm 0.3 \mathrm{~nm}$ \\
& $(318 \pm 16 \mathrm{~L})$ & $(1.8 \pm 0.1 \mathrm{~L})$ & $(43 \pm 2.2 \mathrm{~L})$ \\
\hline \multirow{2}{*}{ S4 } & $36.3 \pm 1.8 \mathrm{~nm}$ & $0.2 \pm 0.01 \mathrm{~nm}$ & $8.2 \pm 0.4 \mathrm{~nm}$ \\
& $(295 \pm 14 \mathrm{~L})$ & $(1.8 \pm 0.1 \mathrm{~L})$ & $(66 \pm 3.3 \mathrm{~L})$ \\
\hline \multirow{2}{*}{ S5 } & $33.6 \pm 1.7 \mathrm{~nm}$ & $0.2 \pm 0.01 \mathrm{~nm}$ & $11.0 \pm 0.6 \mathrm{~nm}$ \\
& $(272 \pm 13 \mathrm{~L})$ & $(1.8 \pm 0.1 \mathrm{~L})$ & $(89 \pm 4.5 \mathrm{~L})$ \\
\hline \multirow{2}{*}{ S6 } & $39.1 \pm 2.0 \mathrm{~nm}$ & $0.2 \pm 0.01 \mathrm{~nm}$ & $5.4 \pm 0.3 \mathrm{~nm}$ \\
& $(318 \pm 15 \mathrm{~L})$ & $(1.8 \pm 0.1 \mathrm{~L})$ & $(43 \pm 2.2 \mathrm{~L})$ \\
\hline \multirow{2}{*}{ S7 } & $39.1 \pm 2.0 \mathrm{~nm}$ & $0.2 \pm 0.01 \mathrm{~nm}$ & $5.4 \pm 0.3 \mathrm{~nm}$ \\
& $(318 \pm 16 \mathrm{~L})$ & $(1.8 \pm 0.1 \mathrm{~L})$ & $(43 \pm 2.2 \mathrm{~L})$ \\
\hline
\end{tabular}

